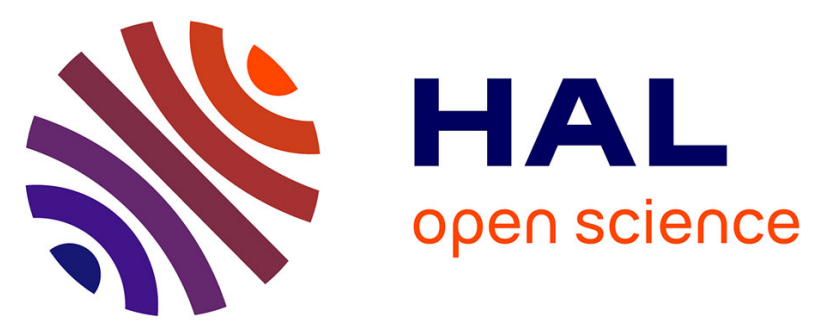

\title{
Thin layers in electrical engineering. Example of shell models in analysing eddy-currents by boundary and finite element methods
}

Laurent Krähenbühl, Daniel Muller

\section{- To cite this version:}

Laurent Krähenbühl, Daniel Muller. Thin layers in electrical engineering. Example of shell models in analysing eddy-currents by boundary and finite element methods. IEEE Transactions on Magnetics, 1993, 29 (2), pp.1450-1455. hal-00082820

\section{HAL Id: hal-00082820 \\ https://hal.science/hal-00082820}

Submitted on 25 Oct 2006

HAL is a multi-disciplinary open access archive for the deposit and dissemination of scientific research documents, whether they are published or not. The documents may come from teaching and research institutions in France or abroad, or from public or private research centers.
L'archive ouverte pluridisciplinaire HAL, est destinée au dépôt et à la diffusion de documents scientifiques de niveau recherche, publiés ou non, émanant des établissements d'enseignement et de recherche français ou étrangers, des laboratoires publics ou privés. 


\title{
Thin layers in electrical engineering. Example of shell models in analysing eddy-currents by boundary and finite element methods
}

\author{
L. Krähenbühl and D. Muller \\ C E G E L Y (URA CNRS N 829) - Ecole Centrale de Lyon - BP 163 \\ F - 69131 Ecully Cedex - (France)
}

\begin{abstract}
During last years, several numerical formulations have been developed by us to model physical problems such as: conducting film effects on the surface of insulators (pollution) [1], high frequency eddy-currents [2], earth field effects on the hull of a ship [3].

The physical effects are completely different, but in each of these examples, they originate from a region thin relative to the other geometrical dimensions. An efficient numerical approach consists of using a surface representation with special boundary conditions expressing the solution inside the thin region.

We propose in this paper a didactical approach to thin regions in electromagnetics and, as an example, the boundary conditions and surface equation for eddy currents flowing inside a thin ferromagnetic shell. The numerical tests are done using the BEM software package PHI3D, but the results could easily be transposed in a FEM context.

The practical applications may concern the computation of losses (shield of electrical machines or transformers) or low frequency electromagnetic perturbations (screen effects, EMC) as well as special applications like optimisation of the induction heating of pans (French art culinaire).
\end{abstract}

\section{INTRODUCTION}

The behaviour of a thin conducting and ferromagnetic shell subjected to a source field depends on frequency and can be analysed analytically.

In the static case, only the ferromagnetic effect appears. The shell attracts the field and channels it, all the more since the permeability of the shell is high. The tangential component of magnetic field is preserved through the shell and obeys on it to a simple differential equation (boundaryequation of continuity).

In the harmonic case, this effect is modified because of eddy currents, which are characterized by the skin depth $\delta$. The type of solution depends essentially on relative values of $e$ (thickness of the shell) and $\delta$. For $e<<\delta$, the static solution remains correct. For $e>>\delta$ the shell is equivalent to a magnetic screen and repels the magnetic field (reverse effect as static); no link subsists locally between field values on both sides of the shell; classical surface impedance or infinite frequency formulations are available.
Between these cases, that is when $e$ and $\delta$ are about equal, all components of flux density or field on both sides of the shell are locally linked through two complicated partial differential boundary equations. The aim of this paper is to build these equations, to expose their physical meaning and to explain how they can be used for numerical computation of fields.

\section{Preliminary Comments}

The developments will be presented here in term of scalar potential or field $\left(\mathbf{H}=\mathbf{H}_{\mathbf{o}}-\operatorname{gradV}\right)$, because it is the most efficient when using the BIEM for the domain equation. Results could be translated easily in terms of vector potential.

\section{II.1. What do we seek?}

The interface conditions used in numerical simulations usually involve the potential $\left(\mathrm{V}_{\mathrm{i}}\right)$ and its normal derivative $\left(d_{n} V_{i}\right)$ on both sides of the interface. To determine these four scalar surface functions we use two domain equations (one for each domain contiguous to the interface) and two interface conditions, which express for example the continuity of tangential field $\left(V_{1}=V_{2}\right)$ and normal flux $\left(d_{n} V_{1}=k \cdot d_{n} V_{2}\right)$.

For the shell problem (Fig. 1) the situation is similar, but the classical interface conditions are to be replaced by relations which take into account the thickness of the shell and its physical behaviour: we will build these relations step by step, from the simplest (magnetostatic) to the most complex ( $e$ and $\delta$ about equal).

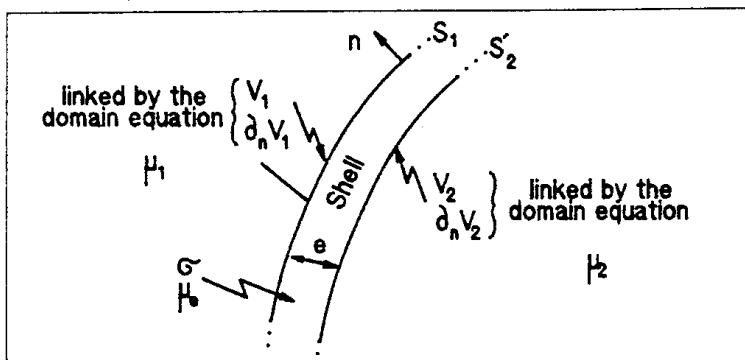

Fig. 1. The thin shell problem: two relations or equations between the boundary values have to be found to describe the internal bebaviour of the shell. 


\section{II.2. Physical interpretation of divergence.}

We said (cf. I) that the magnetostatic property of a ferromagnetic shell is to channel the flux density $B$. What does that mean exactly?

The fundamental property we will use is the continuity of the vector $\mathbf{B}$, mathematically expressed by the equation of continuity:

$$
\operatorname{div} \mathbf{B}=0
$$

This equation expresses the concept of tubes of flux which cannot converge upon a point without again diverging therefrom (Fig. 2a). Some meaning vectors do not have this property: the typical example is the electric induction $D$ :

$$
\operatorname{div} \mathbf{D}=\mathbf{q}
$$

div $D$ is not everywhere equal to zero and it represents the distribution of electric charge (Fig. 2b): in other words, the righ-hand-term of the equation of continuity symbolises the source of the flux of the left-hand-term vector.

\section{II.3. Continuity for a shell.}

Equation (1) takes a particular expression for a shell. To obtain this expression, let us first consider that $B$ is everywhere tangent to the surface of the shell. Then the normal flux is zero and the tangential flux density is divergence free:

$$
\operatorname{div}_{\mathbf{s}} \mathbf{B}_{\mathrm{tg}}=0
$$

In fact, we have to take into account the depth $e$ of the sliell, and the equation of continuity is only valid for the equivalent shell flux density $\mathrm{F}$ defined by the integral value:

$$
\begin{aligned}
& F=\int_{b} B_{s} \cdot d z \\
& \operatorname{div}_{s} F=0
\end{aligned}
$$

On the other hand, what happens when $B$ is not tangent to the surface, that is if we add normal flux densities $B_{1 n}$ and $B_{2 n}$ on both sides $S_{1}$ and $S_{2}$ of the shell? These distributions are
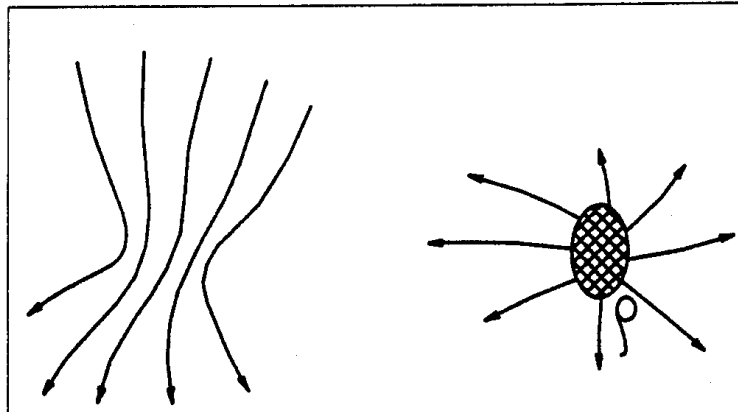

Fig. 2. Typical behaviour of vector-fields [4]:
(a) non-divergent field (e.g. H)
(b) divergent field (e.g. D)

increasing or decreasing the internal flux $F$. Then the resultant flux density $\left(B_{1}-B_{2}\right) \cdot n$ is the actual source of $F$, like $q$ is the source of $\mathbf{D}$ (Fig. 3): then $\mathbf{F}$ becomes divergent:

$$
\operatorname{div}_{s} \mathrm{~F}=\left(\mathbf{B}_{1}-\mathbf{B}_{2}\right) \cdot \mathbf{n}
$$

This is the expression we were looking for. We will now apply this particular form of the equation of continuity to three magnetic or electromagnetic configurations.

\section{Thin Shell, Static}

We consider here the effect of a static magnetic field on a thin ferromagnetic shell, for example the hull of a ship. Since there is no current flowing in the shell, it seems adequate to assign a constant value to the tangential component of $H$ through the depth; then, the shell flux density $F(4)$ can be evaluated;

$$
F=\int_{e} B_{s} \cdot d z=e . B_{s, t}
$$

and the equations for the thin ferromagnetic shell, static, are:

$$
\begin{aligned}
& \mathbf{H}_{\mathrm{tg}, 1}=\mathbf{H}_{\mathrm{tg}, 2} \\
& \text { e } \cdot \mu_{\mathrm{e}} \cdot \operatorname{div}_{\mathrm{s}} \mathrm{H}_{\mathrm{s}}=\mu_{\mathrm{l}} \mathrm{H}_{1, \mathrm{n}}-\mu_{2} \mathrm{H}_{2, \mathrm{n}}
\end{aligned}
$$

If $e . \mu_{e}$ is not a constant, a term $\mathbf{H}_{s} \cdot \operatorname{grad}_{\mathrm{s}} \mathrm{e} \mu_{\mathrm{e}}$ has to be introduced ${ }^{1}$.

The main difference with the regular interface conditions is that $(10)$ is nota direct, algebraic, relation between components of the fields, but a partial differential equation on the surface of the shell, to be solved simultaneously with the domain equations.

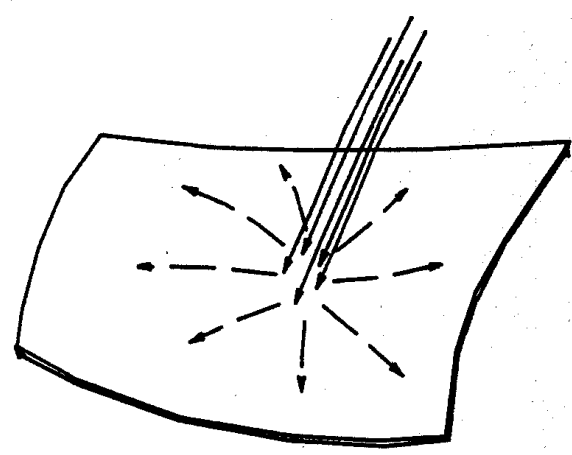

Fig. 3. Flux density in a shell and its source

'Note that, if $e$ goes to zero, the left-hand-term in (10) is zero and we find again the classical interface conditions between two ferromagnetic materials. 


\section{High Frequency EDDY CURRENTS}

We consider here a solid conducting body in a high frequency source field. For a sufficiently high frequency, the field refuses to penetrate deep into the conducting material: because of the skin effect, it looks like a shell.

If the skin deph $\delta$ is small in comparison with the other geometrical dimensions of the conductor, the following well known analytical solution for the penetration of B (Fig. 4) is valid:

$$
\mathbf{B}_{\mathrm{tg}}(\mathrm{z})=\mathbf{B}_{\mathrm{s}} \cdot \mathrm{e}^{-\mathbf{a} \cdot \mathbf{z}} \quad \underline{\mathbf{a}}=(1+\mathbf{j}) / \delta
$$

We can use it to calculate the equivalent flux density $F(4)$ as a function of $B$ on the surface:

$$
\mathbf{F}=(1-\mathrm{j}) / 2 \cdot \delta \cdot \mathbf{B}_{\mathrm{s}, \mathrm{tg}}
$$

This expression is completely analogous to (8), with a complex equivalent depth related to the skin depth:

$$
\underline{e}=\delta \cdot(1-j) / 2
$$

Then, the boundary equations for the high frequency formulation are:

$$
\begin{aligned}
& \mathbf{H}_{2}=\mathbf{0} \\
& \mathrm{e} \cdot \mu_{\mathrm{e}} \cdot \operatorname{div}_{\mathrm{s}} \mathrm{H}_{\mathrm{s}}=\mu_{1} \cdot \mathrm{H}_{1, \mathrm{n}}
\end{aligned}
$$

We find again in a different way the idea of surface impedance [5]. Note the asymptotic solution at very high frequency, when $\delta$ (and e) goes to zero: (15) becomes the simple boudary condition (Neuman, homogenous) of the infinite frequency formulation [6]:

$$
0=\mu_{1} \cdot H_{1, n}
$$

The normal component of field on the conductor vanishes, meaning that the eddy currents constitute a perfect magnetic shield.

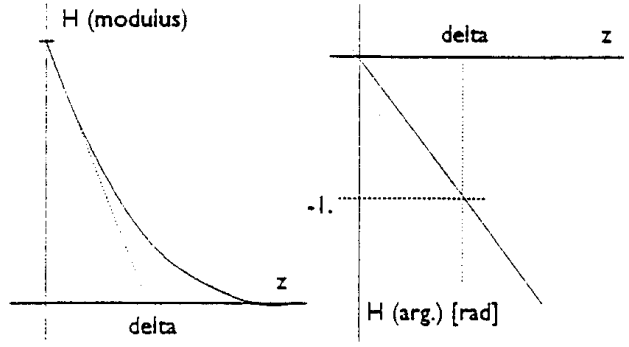

Fig. 4. Skin effect in a solid body

\section{EdDy CuRrents INTO A SHell [7]}

In this case, we have to consider the values of $B$ on both sides of the shell (index 1 and 2). The Maxwell equations, which lead to (11) with a solid body, give the following variation of $\mathbf{B}$ through the shell (Fig. 5):

$$
\mathbf{B}(z)=\mathbf{b} \cdot \mathrm{e}^{-\mathbf{a} \cdot \mathbf{z}}+\mathbf{b}^{\prime} \cdot \mathrm{e}^{+\mathbf{a} \cdot z} \quad \underline{\mathbf{a}}=(1+\mathbf{j}) / \delta
$$

The vector-coefficients $\mathbf{b}$ and $\mathbf{b}^{\prime}$ depend on the surface values $\mathrm{B}_{1}$ and $\mathrm{B}_{2}$.

V.1. First relation: equation of continuity.

The expression of $F(4)$ and the related boundary equation (6) are obtained after some calculations:

$$
\begin{aligned}
& \mathbf{F}=(1-\mathrm{j}) / 2 \cdot \delta \cdot \tanh (\underline{\mathrm{a}} \cdot \mathrm{e} / 2) \cdot\left(\mathrm{B}_{1, \mathrm{tg}}+\mathrm{B}_{2, \mathrm{tg}}\right) \text { and: } \\
& \text { e. } \mu_{\mathrm{e}} \cdot \tanh (\underline{a} \cdot \mathrm{e} / 2) \cdot \operatorname{div}_{\mathrm{s}}\left(\mathrm{H}_{1 \mathrm{~s}}+\mathrm{H}_{2 \mathrm{~s}}\right)=\left(\mu_{1} \cdot \mathrm{H}_{1 \mathrm{n}}-\mu_{2} \cdot \mathrm{H}_{2 \mathrm{n}}\right)
\end{aligned}
$$

The flux flowing into the shell depends on the apparent meanvalue of $\mathrm{B}_{\mathrm{tg}}$, with a corrective factor which takes into account the complex distribution (17). The results (18) and (19) look like (12) and (15) except for the tanh factor, which expresses the shell effect.

The boundary relation corresponding to (9) and (14) becomes more complicated, because the currents flowing in the shell make the tangential field discontinuous. We will first recall some properties of surface-currents.

\section{V.2. Shell current $\boldsymbol{K}$ : general properties.}

Let us define the equivalent shell current $\mathbf{K}$ [8] by:

$$
K=\int_{i} \mathbf{j}(\mathbf{z}) \cdot \mathbf{d z}
$$

Because of the particular direction of $\mathbf{j}$, one has:

$$
\mathbf{j}=\operatorname{curl} \mathbf{H}=-\mathbf{n} \times \partial \mathbf{H}_{\mathrm{tg}} / \partial \mathrm{z}
$$

and:

$$
K=\mathbf{n} \times\left(\mathbf{H}_{1 \mathrm{tg}}-\mathbf{H}_{2 \mathrm{tg}}\right)
$$

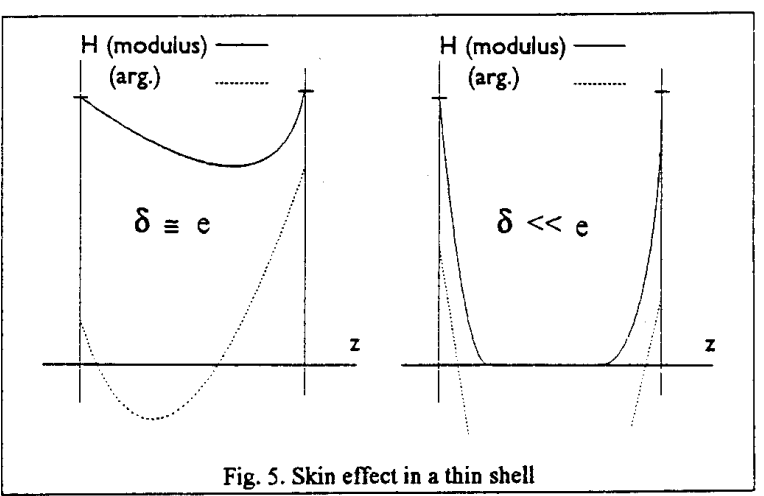




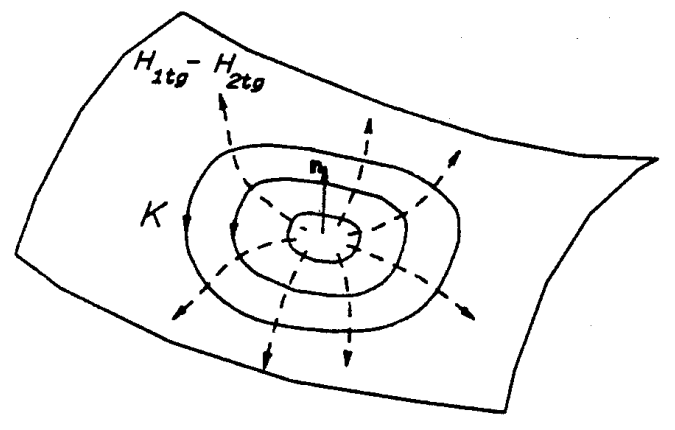

Fig. 6. Shell-current and discontinuity of magnetic field

Then the discontinuity of $\mathrm{H}_{\mathrm{tg}}$ is linked to the surface-current $\mathrm{K}$, meaning that they have same modulus and are perpendicular. As a consequence, when $\mathrm{K}$ curls, the discontinuity of $\mathrm{H}_{t_{8}}$ diverges (Fig. 6):

$$
\text { curl } K=\mathbf{n} \cdot \operatorname{div}_{\mathrm{s}}\left(\mathrm{H}_{1 \mathrm{~s}}-\mathrm{H}_{2 \mathrm{~s}}\right)
$$

V.3. Second relation: Faraday's law for a shell.

We start with Faraday's law and integrate it through the depth.:

$$
\int \operatorname{curl} j \cdot d z=\int-j \omega \sigma \cdot B \cdot d z
$$

The left-hand-term is equivalent to (23). The right-hand-term concerns the normal component of $\mathrm{B}$. The way to calculate it from (17) is the same as we did for $\mathbf{F}$, and the result is similar. Finally we get the particular expression of Faraday's law for a thin shell:

$$
\underline{e} \cdot \mu_{\mathrm{e}} \cdot \operatorname{div}_{\mathrm{s}}\left(\mathrm{H}_{1 \mathrm{~s}}-\mathrm{H}_{2 \mathrm{~s}}\right)=\left(\mu_{1} \cdot \mathrm{H}_{1 \mathrm{n}}+\mu_{2} \cdot \mathrm{H}_{2 \mathrm{n}}\right) \cdot \tanh (\underline{a} \cdot \mathrm{e} / 2)
$$

\section{V.4. Comment.}

Equations (19) and (25) look oddly alike. Only signs are transposed and the tanh factor jumps from the left- to the righthand-term.

The first relation (19) expresses the continuity of flux: it connects the surface variations of the mean value of $\mathrm{H}_{\mathrm{ts}}$ into the shell (i. e. of the shell flux density F) to its source, which is the resultant normal flux density (Fig. 7-a).

The second relation (25) expresses the Faraday's law. It links the surface variations of the field discontinuity (i. e. of the shell current $K$ ) to its source, which is the mean value of the normal flux density through the shell (Fig. 7-b).

Both effects are less or more predominant, depending on relative values of $e$ and $\delta$ (i. e. on value of $q . e$ in the $\tan h$ factor).

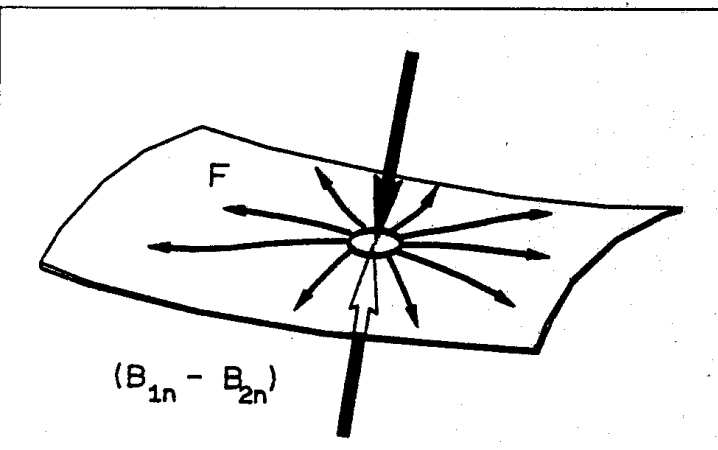

Fig. 7-a. Shell-flux $F$ and its source (19):

$\operatorname{div}\left(\right.$ mean-value of $\left.\mathbf{H}_{\mathbf{L}}\right) \longleftrightarrow$ discontinuity of normal flux density

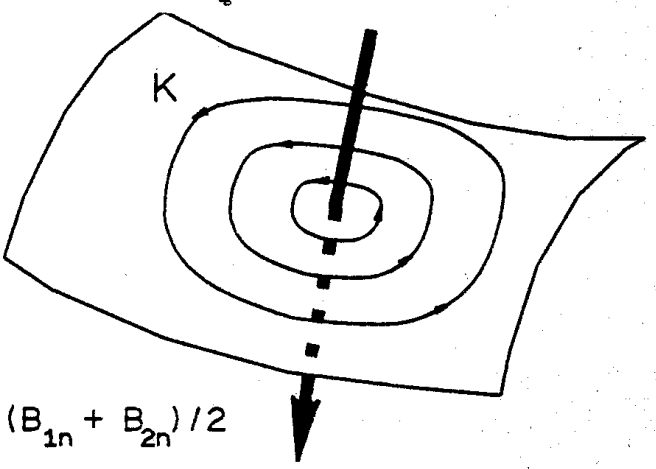

Fig. 7-b. Shell-current $\mathrm{K}$ and its source (25): $\operatorname{div}\left(\right.$ discontinuity of $\mathbf{H}_{\mathrm{QB}}$ ) $<->>$ mean-value of normal flux density

If the frequency goes to zero (i.e. $\delta \gg e$ ), the term $\tanh (q e / 2)$ goes to (qe/2) then:

$$
(18) \Rightarrow F=e \cdot\left(B_{1, t g}+B_{2, t g}\right) / 2
$$

which is to be compared to (6). For high frequencies, the term $\tanh (\underline{a} e / 2)$ goes to 1 , then:

$$
(18)=>F=e \cdot\left(B_{1, t g}+B_{2, t g}\right)
$$

which is to be compared to (12). Equations (19) and (25) give 2 equations like (15), uncoupled, one for each side of the shell.

As a consequence, it becomes apparent that the model of magnetodynamic shell presented here is very general; in particular, it includes both static and high frequency models.

\section{NUMERICAL IMPLEMENTATION.}

The numerical implementation of these shell models naturally depends on the formulation and numerical method used to solve the volumic field problem, outside the shell. They are efficient with variational formulations [3][9] as well as with boundary integral equations. 
We have tested the shell models [1][2][7] in our BIE software package $P H \Pi 3 D[10]$. In that case, the boundary integral equations describing the 3D-domains are to be coupled with the differential boundary equations (10), (15) or (19) and (25) modeling the shell. In both cases, the discretisation uses the same surface finite element mesh, with respectively a point collocation method (BIE) and a variational method (shell). The fields are characterised using the reduced magnetic scalar potential $\mathrm{V}$ :

$$
H=H_{0}-\operatorname{gradV} \quad H_{0}: \text { source field }
$$

\section{VI.1. Equations for a closed shell.}

When the shell surrounds a closed area, it is possible to write two independent BIE (one on each side of the shell), because its sides do not concern the same 3D domain. In that case, the system of equations is (Fig. 1):

$$
\begin{aligned}
& \text { e. } \mu_{\mathrm{e}} \cdot \tanh (\underline{a} \cdot \mathrm{e} / 2) \cdot\left[2 \cdot \operatorname{div}_{\mathrm{S}} \mathrm{H}_{0}-\Delta_{\mathrm{S}}\left(\mathrm{V}_{1}+\mathrm{V}_{2}\right)\right]=-\mathrm{d}_{\mathrm{n}} \mathrm{V}_{1}+\mathrm{d}_{\mathrm{n}} \mathrm{V}_{2} \text { (29) } \\
& \text { e. } \mu_{e} \cdot \Delta_{s}\left(V_{1}-V_{2}\right)=\left[2 \cdot H_{0 n}-\left(d_{n} V_{1}+d_{n} V_{2}\right)\right] \cdot \tanh (a \cdot e / 2) \\
& c_{1} \cdot V_{1}=-\int_{S}\left(V_{1} \cdot d_{n} G-d_{n} V_{1} \cdot G\right) \cdot d s \\
& c_{2} \cdot V_{2}=\int_{S}\left(V_{2} \cdot d_{n} G-d_{n} V_{2} \cdot G\right) \cdot d s
\end{aligned}
$$

\section{VI.2. Equations for an open shell.}

When the shell does not surround a closed area, its sides belong to the same 3D domain. Only one BIE can be written, moreover this equation degenerates because the surfacic integration concerns two surfaces geometrically confused:

(29) and (30) $\Rightarrow$

$$
\left(V_{1}+V_{2}\right) / 2=-\int_{s}\left[\left(V_{1}-V_{2}\right) \cdot d_{n} G-\left(d_{n} V_{1}-d_{n} V_{2}\right) \cdot G\right] \cdot d s
$$

As usual in such a situation [8] we have to differentiate (33) to recover the lost equation. This equation (cf. 36) expresses the mean value of the normal field as an integral function of the discontinuities of $\mathrm{V}$ and $\mathrm{H}_{\mathrm{n}}$.

Finally, we can write all these equations for example in terms of perturbations only, that is, using:

$\mathrm{V}$ : reduced scalar magnetic potential (28): $\mathrm{V}=\left(\mathrm{V}_{1}+\mathrm{V}_{2}\right) / 2$ on the shell

$\phi$ : discontinuity of potential, i.e. current function [8]: $\phi=\left(V_{1}-V_{2}\right)$ with the properties:

$\mathbf{K}=-\mathbf{n} \times \operatorname{grad}_{\mathrm{S}} \phi \quad$ or: $\left(\mathbf{H}_{1}-\mathbf{H}_{2}\right)_{\mathrm{tg}}=-\operatorname{grad}_{\mathrm{s}} \phi$

The iso- $\phi$ are the lines of the shell-current $K$.

$\theta$ : discontinuity of the normal field, i.e. source of $V$ : $\theta=\mathrm{H}_{1 \mathrm{n}}-\mathrm{H}_{2 \mathrm{n}}=-\left(\mathrm{d}_{\mathrm{n}} \mathrm{V}_{1}-\mathrm{d}_{\mathrm{n}} \mathrm{V}_{2}\right)$

$\psi:$ modification of the mean-value of the normal component of field, i.e. source of $\phi$. $\psi=\left(\mathrm{H}_{1 \mathrm{n}}+\mathrm{H}_{2 \mathrm{n}} / 2-\mathrm{H}_{0 \mathrm{n}}=-\left(\mathrm{d}_{\mathrm{n}} \mathrm{V}_{1}+\mathrm{d}_{\mathrm{n}} \mathrm{V}_{2}\right) / 2\right.$
One obtains the following system of equations:

$$
\begin{aligned}
& \text { e. } \mu_{\mathrm{e}} \cdot \operatorname{th}(\mathrm{a} \cdot \mathrm{e} / 2) \cdot\left(\operatorname{div}_{\mathrm{S}} \mathrm{H}_{0}-2 \cdot \Delta_{\mathrm{s}} \mathrm{V}\right)=\theta \\
& \text { e. } \mu_{\mathrm{e}} \cdot \Delta_{\mathrm{s}} \phi=2 \cdot\left(\psi+\mathrm{H}_{0 \mathrm{n}}\right) \cdot \operatorname{th}(\mathrm{a} \cdot \mathrm{e} / 2) \\
& \psi=-\mathbf{n} \cdot \int_{\mathrm{s}}\left[\left(\operatorname{mxgrad}_{\mathrm{s}} \phi\right) \times \operatorname{grad} \mathrm{G}-\theta \cdot \operatorname{gradG}\right] . \mathrm{ds} \\
& \mathrm{V}=-\int_{\mathrm{s}}(\phi \cdot \mathrm{m} \cdot \operatorname{grad} \mathrm{G}-\theta \cdot \mathrm{G}) \cdot \mathrm{ds}
\end{aligned}
$$

where: $\mathbf{n}$ is the normal vector on the point of collocation, $\mathbf{m}$ is the normal vector on the point of integration.

\section{NUMERICAL TESTS.}

The general magnetodynamic shell model (Eq. 29 to 32, or 34 to 37) has not yet been used to solve an industrial problem, at least with measurements of good quality.

However, the other shell models presented above have already proved their efficiency and their accuracy. The static formulation is successfully used, in particular to evaluate the magnetisation of ships due to the earth field. Excellent results were also obtained for the TEAM Workshop problem \#13 [11][12], where the object of interest is made of thin ferromagnetic plates $(3.2 \mathrm{~mm}$ thick, other dimensions: 50 to $120 \mathrm{~mm}$ ).

High frequency formulations (surface impedance and infinite frequency limit) have also been used for quite some time for NDT and induction heating simulations. The coupling with computation of thermal effects and the progress in modelling cracks will promote these models.

\section{Magnetodynamics: comparison with analytical results.}

The first step to validate a new formulation is to compare the solution it gives with analytical results. We will considere here a hollow sphere in an alternating source field (Fig. 10). This is a simple configuration, but both ferromagnetic and eddy current effects are clearly shown (Fig. 11): it has been solved successively using the static formulation $\{S F,(9)$ and $(10)\}$, the high frequency formulation $\{H F F,(15)\}$, the infinite frequency formulation $\{I F F,(16)\}$ and the general magnetodynamic formulation $\{G F,(29)$ to (32) $\}$ with a minimal mesh (6 elements, 27 nodes, for $1 / 8$ sphere).

Results on test-point $P$ are summarised on Fig. 11. Analytical and numerical results are indistinguishable: in fact the error is less than $1 \%$, that is the extent of the error of discretisation. 

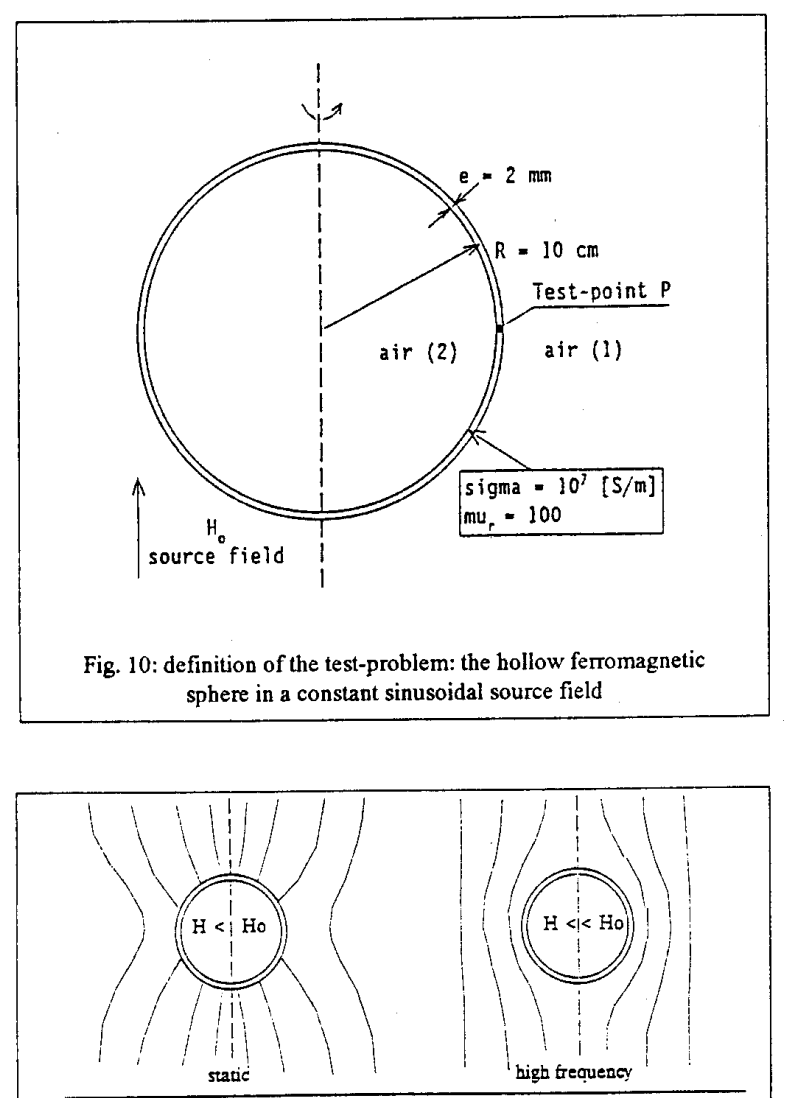

Fig. 3: Fiux lines piot (scherratic)

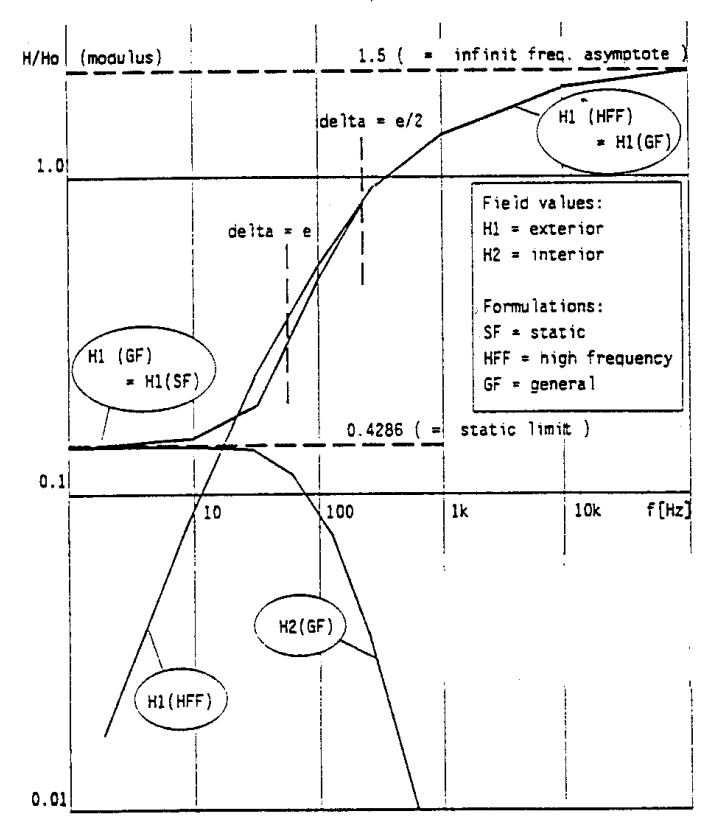

Fig. 11: the hollow sphere test-problem: field modulus on $\mathrm{P}$ computed with 3 different shell formulations

\section{CONCLUSION}

With given geometrical dimensions and physical properties, we can see that:

- for $\mathrm{f}<1 \mathrm{~Hz}, \mathrm{SF}$ and GF give the same result.

- for $\mathrm{f}>250 \mathrm{~Hz}$ (i.e. $\delta<e / 2$ ) GF and HFF are equivalent.

- GF allows the exact computation of the passage from static to high frequency.

We have to stress the price to pay: for the same mesh, the magnetodynamic general formulation (GF) needs 8 times as many real unknowns as the infinit frequency formulation (IFF), 4 times as many as the static formulation (SF) and 2 times as many as the high frequency formulation: it has to be used with discrimination! Still it remains extremely efficientin comparison with a finite element mesh of the volume of the shell.

The research continues, to take into account more and more complex phenomenas (saturation, anisotropy, multi-layers shell, etc.). We will also set up some experiments to valid the shell models within industrial configurations.

\section{REFERENCES}

[1] J.L. Rasolonjanahary, L. Krähenbühl,A. Nicolas, "Computation of electric fields and potential on polluted insulators using a boundary elemen method," IEEE Trans. on Mag.., vol. $28 N^{\circ} 2$, March 92, pp. 1473-1476.

[2] L. Krähenbühl,"Surface current and eddy-current $3 D$ computation using BIE tehniques," Proc. of the $3 \mathrm{rd}$ int. IGTE symposium, pp. 40-50. Graz, Austria, Sept. 88.

[3] X.Brunotte, G. Meunier, "Hybrid finite element/boundary equationmethod for the analysis of magnetic field created by complex geometries in an uniform external field," Proc. of the int. boundary element Symp., Nice. France, 15-17 mai 1990 - Comput. Mech. Publication, Springer-Verlag..

[4] B. Hague, "Electromagnetic Problems in Electrical Engineering," Oxford University Press, 1929 - Dover Publications, Inc, New-York, 1962.

[5] T.H. Fawzi, M. Taher Ahmed, P.E. Burke, "On the use of the impedance boundary conditions in eddy-durrent problems," IEEE Trans. on Mag., vol. 21, Sept. 85, pp. 1835-1840.

[6] B. Ancelle, A. Nicolas, J.C. Sabonnadière, "A boundary integral equation method for high frequency eddy-currents," IEEE Trans. on Magn., vol. 17 $n^{\circ} 6, p p .2568-2570$, Nov. 1981 .

[7] L. Krähenbühl, "A theory of thin layers in Electrical Engineering. Application to eddy-current calculation inside a shell using the BIE software PHI3D," Proc. of the 4th int. IGTE symposium and european TEAM Workshop, pp. 101-106, Graz, Austria, Oct. 90.

[8] E. Durand, "Magnetostatique, (chapitre II)" Masson et Cie, Paris, 1968.

[9] J.P. Sturgess, T.W. Preston, "An economic solution for 3-D coupled electromagnetic and thermal eddy current problems," IEEE Trans. on Mag., vol. $28 N^{\circ} 2$, March 92, pp. 1267-1270.

[10] L. Krähenbühl, A. Nicolas, L. Nicolas, "The CAD package PHI3D for the computation of electric or magnetic fields in 3D devices," Compel, vol. 9 . suppl. A, pp. 185-189.

(11] "3DMAG Abstracts," Proc. of the intern. symposium and TEAM Workshop. on 3-D electromagneticfield analysis, Sept.11-13, 1989, Okayama, Japan.

[12] Proc. of the 4th int. IGTE symposium and eirropean TEAM Workshop, Graz, Austria, Oct. 90. 